\title{
A murine lung cancer co-clinical trial identifies genetic modifiers of therapeutic response
}

\author{
Zhao Chen ${ }^{1,2,3}$, Katherine Cheng 2,3 , Zandra Walton 2,3 , Yuchuan Wang ${ }^{4,5,6}$, Hiromichi Ebi ${ }^{1,7}$, \\ Takeshi Shimamura ${ }^{8}$, Yan Liu' ${ }^{1,2,3}$, Tanya Tupper ${ }^{4}$, Jing Ouyang ${ }^{2}$, Jie Li ${ }^{9}$, Peng Gao ${ }^{2,3}$, \\ Michele S. Woo $^{2}$, Chunxiao $\mathbf{X u}^{1,2,3}$, Masahiko Yanagita ${ }^{2}$, Abigail Altabef ${ }^{2}$, Shumei Wang ${ }^{10}$, \\ Charles Lee ${ }^{10}$, Yuji Nakada ${ }^{11}$, Christopher G. Peña ${ }^{11}$, Yanping Sun ${ }^{4,5}$, Yoko Franchetti ${ }^{12}$, \\ Catherine $\mathrm{Yao}^{2}$, Amy Saur ${ }^{4}$, Michael D. Cameron ${ }^{13}$, Mizuki Nishino ${ }^{5,6}$, D. Neil Hayes ${ }^{14}$, \\ Matthew D. Wilkerson ${ }^{14}$, Patrick J. Roberts ${ }^{14}$, Carrie B. Lee ${ }^{14}$, Nabeel Bardeesy ${ }^{7}$, Mohit \\ Butaney ${ }^{2}$, Lucian R. Chirieac ${ }^{10}$, Daniel B. Costa ${ }^{15}$, David Jackman ${ }^{2}$, Norman E. \\ Sharpless ${ }^{14}$, Diego H. Castrillon ${ }^{11}$, George D. Demetri ${ }^{3}$, Pasi A. Jänne ${ }^{1,2,16}$, Pier Paolo \\ Pandolfi ${ }^{17}$, Lewis C. Cantley ${ }^{18,19}$, Andrew L. Kung ${ }^{4,20}$, Jeffrey A. Engelman ${ }^{1,7}$, and Kwok- \\ Kin Wong ${ }^{1,2,3,16}$ \\ ${ }^{1}$ Department of Medicine, Harvard Medical School, Boston, Massachusetts 02115, USA \\ 2Department of Medical Oncology, Dana-Farber Cancer Institute, Boston, Massachusetts 02115, \\ USA \\ ${ }^{3}$ Ludwig Center at Dana-Farber/Harvard Cancer Center, Dana-Farber Cancer Institute, Boston, \\ Massachusetts 02115, USA \\ ${ }^{4}$ Lurie Family Imaging Center, Dana-Farber Cancer Institute, Boston, Massachusetts 02115, USA \\ ${ }^{5}$ Department of Imaging, Dana-Farber Cancer Institute, Boston, Massachusetts 02115, USA \\ ${ }^{6}$ Department of Radiology, Brigham and Women's Hospital, Boston, Massachusetts 02115, USA \\ ${ }^{7}$ Department of Medical Oncology, Massachusetts General Hospital Cancer Center, Boston, \\ Massachusetts 02114, USA \\ ${ }^{8}$ Department of Molecular Pharmacology and Therapeutics, Oncology Institute, Loyola University \\ Chicago Stritch School of Medicine, Maywood, Illinois 60153, USA \\ ${ }^{9}$ Center for Survey Statistics \& Methodology, Department of Statistics \& Statistical Laboratory, \\ lowa State University, Ames, lowa 50010, USA \\ ${ }^{10}$ Department of Pathology, Brigham and Women's Hospital, Boston, Massachusetts 02115, USA \\ ${ }^{11}$ Department of Pathology and Simmons Comprehensive Cancer Center, UT Southwestern \\ Medical Center, Dallas, Texas 75390, USA
}

(C) 2012 Macmillan Publishers Limited. All rights reserved.

Correspondence and requests for materials should be addressed to K.-K.W. (kwong1 @ partners.org), J.A.E. (jengelman@ partners.org) or A.L.K. (andrew_kung@dfci.harvard.edu).

Supplementary Information is linked to the online version of the paper at www.nature.com/nature.

Author Contributions Z.C., K.C., Z.W., Y.W., H.E., T.S., Y.L., T.T., J.O., J.L., P.G., M.S.W., C.X., M.Y., A.A., S.W., C.L., Y.N., C.G.P., Y.S., Y.F., C.Y., A.S., M.D.C., D.N.H., M.D.W., P.J.R., C.B.L., N.B., N.E.S., D.H.C., G.D.D., P.A.J., L.C.C., C.B.L., M.N and P.P.P. performed experimental work and data analyses. M.B., L.R.C., D.B.C. and D.J. collected data and provided patient materials. A.L.K., J.A.E. and K.-K.W. conceived and supervised all aspects of the project. All authors contributed to the final manuscript.

Author Information Reprints and permissions information is available at www.nature.com/reprints. The authors declare competing financial interests: details accompany the full-text HTML version of the paper at www.nature.com/nature. Readers are welcome to comment on the online version of this article at www.nature.com/nature. 
${ }^{12}$ Department of Biostatistics and Computational Biology, Dana-Farber Cancer Institute, Department of Biostatistics, Harvard School of Public Health, Boston, Massachusetts 02115, USA

${ }^{13}$ Translational Research Institute, The Scripps Research Institute, Jupiter, Florida 33458, USA

${ }^{14}$ The Lineberger Comprehensive Cancer Center, University of North Carolina, Chapel Hill, North Carolina 27599, USA

${ }^{15}$ Division of Hematology/Oncology, Beth Israel Deaconess Medical Center, Harvard Medical School, Boston, Massachusetts 02115, USA

${ }^{16}$ Lowe Center for Thoracic Oncology, Dana-Farber Cancer Institute, Boston, Massachusetts 02115, USA

${ }^{17}$ Cancer Genetics Program, Beth Israel Deaconess Cancer Center, Department of Medicine and Pathology, Beth Israel Deaconess Medical Center, Harvard Medical School, Boston, Massachusetts 02115, USA

${ }^{18}$ Department of Systems Biology, Harvard Medical School, Boston, Massachusetts 02115, USA

${ }^{19}$ Division of Signal Transduction, Beth Israel Deaconess Medical Center, Boston, Massachusetts 02115, USA

20Department of Pediatric Oncology, Dana Farber Cancer Institute and Children's Hospital, Boston, Massachusetts 02115, USA

\section{Abstract}

Targeted therapies have demonstrated efficacy against specific subsets of molecularly defined cancers ${ }^{1-4}$. Although most patients with lung cancer are stratified according to a single oncogenic driver, cancers harbouring identical activating genetic mutations show large variations in their responses to the same targeted therapy ${ }^{1,3}$. The biology underlying this heterogeneity is not well understood, and the impact of co-existing genetic mutations, especially the loss of tumour suppressors $^{5-9}$, has not been fully explored. Here we use genetically engineered mouse models to conduct a 'co-clinical' trial that mirrors an ongoing human clinical trial in patients with $K R A S$ mutant lung cancers. This trial aims to determine if the MEK inhibitor selumetinib (AZD6244) ${ }^{10}$ increases the efficacy of docetaxel, a standard of care chemotherapy. Our studies demonstrate that concomitant loss of either $p 53$ (also known as $T p 53$ ) or $L k b 1$ (also known as Stk11), two clinically relevant tumour suppressors $6,9,11,12$, markedly impaired the response of Kras-mutant cancers to docetaxel monotherapy. We observed that the addition of selumetinib provided substantial benefit for mice with lung cancer caused by Kras and Kras and p53 mutations, but mice with Kras and $L k b 1$ mutations had primary resistance to this combination therapy. Pharmacodynamic studies, including positron-emission tomography (PET) and computed tomography (CT), identified biological markers in mice and patients that provide a rationale for the differential efficacy of these therapies in the different genotypes. These co-clinical results identify predictive genetic biomarkers that should be validated by interrogating samples from patients enrolled on the concurrent clinical trial. These studies also highlight the rationale for synchronous co-clinical trials, not only to anticipate the results of ongoing human clinical trials, but also to generate clinically relevant hypotheses that can inform the analysis and design of human studies.

Activating $K R A S$ mutations are found in $15-30 \%$ of all patients with non-small cell lung cancer (NSCLC), and predict poor outcome in response to conventional treatment regimens ${ }^{13,14}$. Preclinical studies have suggested that inhibition of MAPK/ERK kinase (MEK) may be effective against $K R A S$-mutant $\mathrm{NSCLC}^{15}$, prompting an ongoing human clinical trial comparing docetaxel monotherapy (standard of care) to docetaxel combined with the MEK inhibitor selumetinib (AZD6244). Although the sole genetic entry criteria for 
patients on this trial is the presence of KRAS mutations, the complexity of NSCLC dictates that many tumours will harbour concomitant genetic alterations that may modulate response to therapy. To mirror this human clinical trial in a murine co-clinical trial, and to investigate the modulating effects of concomitant tumour suppressor loss, we generated cohorts of genetically engineered mice with either Kras, Kras and $p 53$ (Kras/p53) or Kras/Lkb1 mutant lung cancers. Activation of $\operatorname{Kras}(G 12 D)$ and inactivation of $p 53$ or $L k b 1$ in the lung epithelium was achieved using nasal instillation of adenovirus encoding the CRE recombinase ${ }^{7}$. Mice with established disease, defined by tachypnoea, hypoxaemia on pulse oximetry ${ }^{16}$, and bulk disease on magnetic resonance imaging (MRI) ${ }^{17}$, were randomized to receive either docetaxel $16 \mathrm{mg} \mathrm{kg}^{-1}$ every other day by intraperitoneal injection ${ }^{18}$ (Supplementary Table 1), selumetinib at $25 \mathrm{mg} \mathrm{kg}^{-1}$ daily by oral gavage ${ }^{19}$, or docetaxel in combination with selumetinib. Treatment response was determined by serial MRI. Tumour volumes were reconstructed from the MRI images (Supplementary Fig. 1a) with a high level of interoperator reliability (Supplementary Fig. 1b; 95\% confidence interval, $-25.6 \%$ to $+31.4 \%)$. On the basis of these performance metrics, and paralleling human response criteria, we used a threshold of $30 \%$ change in tumour volume to define progressive disease and partial response.

For tumours with only Kras mutation, treatment with docetaxel monotherapy resulted in a modest rate of response, with $30 \%$ of mice achieving a partial response (Fig. 1a, c). Mice bearing Kras tumours with concurrent loss of $p 53$ or $L k b 1$ had markedly lower response rates to docetaxel treatment (5\% and $0 \%$, respectively), and more of these animals demonstrated progressive disease on MRI or progression to moribundity (Fig. 1a and Supplementary Table 2). The addition of selumetinib to docetaxel treatment provided substantial benefit (Fig. 1b, c), with the overall response rate increased to $92 \%$ in Krasmutant cancers $\left(P=2.8 \times 10^{-5}\right.$, Fisher exact test compared to docetaxel alone) and $61 \%$ in Kras/p53 mice $\left(P=2.7 \times 10^{-4}\right)$. In contrast, for $K r a s / L k b 1$ mutant cancers the addition of selumetinib to docetaxel led to only a modest improvement in overall response, with $33 \%$ of the mice achieving a partial response (Fig. 1b, c). Compared to the other genotypes, Kras/ $L k b 1$ mice had a significantly lower rate of response to combined treatment with selumetinib and docetaxel $(P=0.0009,3 \times 2$ contingency Fisher exact test $)$.

The magnitude of change in volume confirmed that tumours with Kras or Kras/p53 mutations were considerably more responsive to combination therapy compared to docetaxel alone. In contrast, the addition of selumetinib did not significantly reduce the volume of tumours with compound Kras/Lkb1 mutations (Fig. 1d and Supplementary Fig. 1c, d). Concordantly, histopathological assessments of tumours collected after two doses of treatment revealed that the combination treatment increased apoptosis (Fig. 1e and Supplementary Fig. 2a) and reduced proliferation(Fig. 1f and Supplementary Fig. 2b) in the Kras and Kras/p53 tumours compared to docetaxel alone, but this was not observed in Kras/ Lkb1 tumours (Fig. 1e, f and Supplementary Fig. 2a, b). These results demonstrate that combined treatment with selumetinib and docetaxel induces apoptosis and decreases proliferation in Kras and Kras/p53 tumours, leading to antitumour efficacy, but that concomitant mutation of $L k b 1$ confers primary resistance to the combination treatment.

Because repeated tumour biopsies are difficult in patients, we explored the use of ${ }^{18} \mathrm{~F}$ fluoro-2-deoxy-glucose PET (FDG-PET) as an early response indicator that could be used in the clinic. Comparison of FDG avidity, quantified by standardized uptake value (SUV) ${ }^{20,21}$ in lung cancers across the three different genotypes showed an overall higher FDG uptake in both Kras/p53 and Kras/Lkb1 tumours compared to Kras tumours (Fig. 2a; $P=0.02$, oneway ANOVA). Expression of the glucose transporter GLUT1 (also known as SLC2A1) was elevated in Kras/Lkb1 mutant tumours (Supplementary Fig. 3a), consistent with the increased baseline FDG-PET signal. To determine if this finding was applicable to human 
patients, we determined the pre-treatment FDG avidity in nine patients with $K R A S$-mutated lung cancer. Tumours from three patients positive for LKB1 immunostaining had a mean maximum SUV ( $\left(\mathrm{SUV}_{\max }\right)$ of 2.33, whereas tumours from six patients negative for LKB1 immunostaining had a mean $\mathrm{SUV}_{\max }$ of 8.75 (Fig. 2b; $P=0.048$, two-sided Wilcoxon).

We next used FDG-PET to assess early tumour metabolic changes after initiation of therapy. Treatment with docetaxel alone did not result in significant changes in tumour hypermetabolism in Kras-, Kras/p53-or Kras/Lkb1-tumour-bearing mice (Fig. 2c, d). Of note, some of the murine Kras lung cancer nodules were not FDG avid (Fig. 2a) and these were the most sensitive to single-agent docetaxel (data not shown). In contrast, within $24 \mathrm{~h}$ of the first dose of treatment with docetaxel and selumetinib, tumour hypermetabolism was markedly suppressed in both Kras and Kras/p53 mice (Fig. 2c, d). However, Kras/Lkb1mutant tumours had no appreciable decrease in FDG avidity when treated with the combination (Fig. 2c, d). Together, these results demonstrate that early changes in tumour metabolism measured by FDG-PET (Fig. 2c, d) are concordant with histopathological analysis of apoptosis and proliferation (Fig. 1e, f) and predict antitumour efficacy (Fig. 1ac) of docetaxel and selumetinib in treating Kras-mutant lung cancers.

To assess the pharmacodynamic effects of treatment on the MEK-ERK signalling axis, we assayed pathway activation using phospho-ERK immunostaining of lung cancer nodules. At baseline, the ERK pathway was most activated in Kras/p53-mutant tumours (Fig. 3a, b). We observed substantially less phospho-ERK staining in Kras/Lkb1 tumours, suggesting that the MEK-ERK pathway is not highly activated in these cancers. Treatment with docetaxel did not alter phospho-ERK staining, but, as expected, the addition of selumetinib decreased MEK-ERK signalling in the Kras and Kras/p53 tumours (Fig. 3a, b).

We further evaluated cellular signalling from short-term-treated lung cancer nodules by immunoblotting tumour lysates. Concordant with immunostaining (Fig. 3a), elevated phospho-ERK and phospho-90RSK were observed in Kras/p53 tumours relative to the other genotypes (Fig. 3c and Supplementary Fig. 4a). Kras/Lkb1 tumours displayed low basal activation of the MEK-ERK pathway (Fig. 3c and Supplementary Fig. 4a), consistent with immunostaining (Fig. 3a). Treatment with docetaxel had no discernable impact on the MEK-ERK pathway in any genotype (Fig. 3a-c and Supplementary Fig. 4b). Although selumetinib alone resulted in decreased phospho-ERK, residual activity was still present (Fig. 3c and Supplementary Fig. 4b). Treatment with both docetaxel and selumetinib more effectively eradicated phospho-ERK activity (Fig. 3c and Supplementary Fig. 4b).

Pharmacokinetic studies suggested that selumetinib levels were elevated in the serum and tumours of mice treated with selumetinib combined with docetaxel compared to selumetinib alone (Supplementary Table 3), perhaps providing a mechanism for the more potent suppression of MEK-ERK signalling by the combination (Fig. 3c). The potential relevance of these findings to human disease was investigated by assessing phospho-ERK staining in a set of 57 human NSCLC tumour samples with known KRAS, p53 and LKB1 mutation status. Consistent with our findings in murine tumours, of seven patients harbouring the $K R A S$ activating mutation, the three patients with concurrent $p 53$ loss showed higher phospho-ERK activity (Fig. 3d).

The decreased activation of ERK phosphorylation in Kras/Lkb1 tumours suggests that the proliferation of these tumours may be driven through other signalling pathways. On the basis of our prior studies ${ }^{19,22}$, we investigated the activity of AKT and SRC in Kras/Lkb1 mutant tumours. Immunoblotting with activation-state-specific antibodies revealed that Kras/Lkb1-mutant tumours have heightened activation of both AKT and SRC (Supplementary Fig. 3a, b), consistent with the finding of increased FDG avidity in Kras/ Lkb1 tumours (Fig. 2a, b), because PI3K regulates expression of GLUT1 (Supplementary 
Fig. 3a). These results suggest that concomitant mutation of Kras and $L k b 1$ may alter the signalling circuitry in tumour cells from one dependent on MEK-ERK (in Kras and Kras/ $p 53$ tumours) to one that has more active AKT and SRC pathways, resulting in primary resistance to docetaxel and selumetinib.

The concurrent human clinical trial does not include a treatment arm in which patients are treated with selumetinib alone, based on lack of efficacy in a phase II clinical trial in patients with NSCLC ${ }^{23}$, and on our preclinical data in Kras genetically engineered mice ${ }^{19}$. In mice with Kras, Kras/p53 and Kras/Lbk1 tumours, treatment with selumetinib alone resulted in a heterogeneous reduction in FDG-PET uptake (Supplementary Fig. 5a), consistent with pharmacodynamic evidence that selumetinib alone partially attenuates MEK-ERK signalling within tumours (Fig. 3c and Supplementary Fig. 7c). However, no partial responses were achieved in any genotype with selumetinib monotherapy, although there was attenuation of tumour growth compared to untreated controls (Supplementary Fig. 5b). Together, these data suggest that selumitinib as monotherapy modulates MEK-ERK signalling in Kras-driven tumours, but is insufficient for clinical benefit in mice and humans.

We determined the long-term benefit of combined treatment with docetaxel and selumetinib in the Kras- and Kras/p53-mutant mice compared to chronic treatment with docetaxel monotherapy. We did not assess long-term treatment outcome in Kras/Lkb1 animals given the primary resistance to both treatments in these animals (Figs 1-3). In mice with Kras tumours, treatment with docetaxel alone stabilized disease for several weeks, whereas the addition of selumetinib caused frank tumour regression and slower tumour re-growth (Fig. 4a and Supplementary Fig. 6a, b). Accordingly, the addition of selumetinib to docetaxel significantly prolonged progression-free survival (Fig. 4b). In mice with Kras/p53 tumours, treatment with docetaxel alone largely resulted in progressive disease, whereas animals treated with a combination of docetaxel and selumetinib had initial disease regression before progression (Fig. 4a and Supplementary Fig. 6c), resulting in prolonged progression-free survival (Fig. 4c). These results demonstrate that the enhanced response to treatment with combined therapy translates to improved progression-free survival, albeit not outright cure, in mice bearing Kras- and Kras/p53-mutant tumours.

To investigate mechanisms of resistance upon disease progression, tumour nodules were isolated from moribund animals after long-term treatment with docetaxel and selumetinib. In all animals examined (5/5 in Kras/p53 and 11/11 in Kras), tumour nodules showed recrudescence of ERK phosphorylation (Fig. 4d and Supplementary Fig. 7a), suggesting that acquired resistance could be partly due to reactivation of MEK-ERK signalling despite ongoing treatment with selumetinib. We evaluated treatment-resistant nodules for ERK amplification (Supplementary Fig. 7b), activation of parallel signalling pathways (Supplementary Fig. 7c), and drug pharmacokinetics (Supplementary Fig. 7d), and did not find consistent changes, suggesting more than one mechanism for pathway reactivation. Efforts to identify the diversity of mechanisms responsible for acquired resistance are ongoing.

This co-clinical study provides several insights and predictions that affect the interpretation of the concurrent human clinical trial. First, these results predict that combination therapy with docetaxel and selumetinib will be more effective than docetaxel alone in several subclasses of KRAS-mutant NSCLC. These data are consistent with the results of the human phase II clinical trial described in a recent press release (http://phx.corporate-ir.net/phoenix.zhtml?c=123810\&p=irol-newsArticle\&ID=1611800). However, our studies predict that concurrent mutation of $L K B 1$ will confer primary resistance to combination therapy, possibly through activation of parallel signalling pathways such as AKT and SRC. As $L K B 1$ status is not being prospectively assessed in the 
ongoing human clinical trial, inclusion of patients with cancers harbouring concurrent $L K B 1$ mutations may blunt differences between treatment arms based solely on $K R A S$ status. These results suggest that a retrospective analysis of $p 53$ and $L K B 1$ status in samples from the concurrent human clinical trial is warranted, and lead us to advocate prospective analysis in future clinical trials with sufficient enrolment to all strata to enable sufficiently powered sub-group analyses.

Beyond assessing genetic modulators, co-clinical studies allow for validation of biomarker strategies and discovery of mechanisms of resistance that may benefit future clinical trials. In this study, we observed the potential utility of FDG-PET imaging as a biomarker strategy for identifying an enriched responder population and predicting long-term outcome. Prior studies have suggested that lung tumour hypermetabolism at the time of diagnosis predicts poor outcome in response to conventional therapies ${ }^{20,24}$, our current study further suggests that high baseline FDG avidity may predict poor response to targeted therapy with selumetinib combined with docetaxel. Specifically, loss of LKB1 function appears to confer increased FDG avidity, probably through upregulated expression of glucose transporters. As current approaches for assessing LKB1 status are not comprehensive, FDG-PET imaging may represent a practicable patient stratification strategy. Furthermore, the current preclinical study suggests a role for repeat FDG-PET imaging early in the course of treatment as a potential predictor of outcome, as metabolic changes may be apparent within $24 \mathrm{~h}$ of initiating therapy. In these studies, we also observed reactivation of the MEK-ERK signalling pathway in mice that became resistant to the combination of selumetinib and docetaxel. Although the exact mechanisms responsible for pathway reactivation remain to be elucidated, mechanisms of resistance discovered in co-clinical studies should be confirmed in human clinical trials by examining biopsy samples from patients who relapse on therapy. The ability to assess mechanisms of resistance in the preclinical setting may uncover rational combinatorial strategies that can be implemented in future clinical studies.

Building upon prior success using genetically engineered mouse models ${ }^{25-27}$, the current study demonstrates that co-clinical trials can provide data that has value beyond predicting the outcome of clinical trials, and can rapidly generate new clinically relevant hypotheses that can affect how the concurrent human clinical trial is analysed, and inform the design of future clinical studies. As similar efforts are undertaken in other cancer disease types, we anticipate that murine co-clinical trials will enable more effective oncology drug development.

\section{METHODS SUMMARY}

\section{Mice}

Mouse strains harbouring a conditional activating mutation (G12D) at the endogenous Kras locus, conditional $L k b 1$ knockout, and conditional p53 knockout were described previously ${ }^{7}$. Genotypes were confirmed by PCR (Supplementary Fig. 8). All studies were performed on protocols approved by Dana-Farber Cancer Institute and University of North Carolina Animal Care and Use Committees, and all mice used are listed in Supplementary Table 4.

\section{MRI quantification}

3D Slicer was used to reconstruct MRI volumetric measurements ${ }^{28,29}$ (Supplementary Fig. 1a). To assess variation between independent operators, Bland-Altman analysis was performed using quantification results from the two operators on a total of 16 MRI scan images (Supplementary Fig. 1b). 


\section{Pharmacokinetics}

Docetaxel concentrations in serum, lung, and tumour nodules was determined using published doses $\left(8\right.$ and $16 \mathrm{mg} \mathrm{kg}^{-1}$ ). Drug concentration was determined $3 \mathrm{~h}$ after the last dose (Supplementary Table 1). All serum concentrations were within the range found in the clinical setting ${ }^{30}$. Selumetinib was administered as previously described ${ }^{19}$, and pharmacokinetics in mice were also documented (Supplementary Table 3).

\section{PET/CT studies}

All murine FDG-PET/CT studies were performed with a pre-clinical small animal PET/CT system (Siemens Inveon) after injection with $14 \mathrm{MBq}$ of ${ }^{18}$ F-FDG. Mice used for PET/CT studies are listed in Supplementary Table 5.

\section{Human samples and clinical information}

All human samples and clinical information were obtained under Institutional Review Board approved protocols (02-180 and 07-0120), and patient information is listed in Supplementary Tables 7 and 8 . A tissue microarray (TMA) was generated from genotyped human lung cancer samples as previously described ${ }^{7}$. The TMA was immunostained for phospho-ERK and scored by a pathologist blinded to patient information.

\section{Immunohistochemistry staining}

Immunohistochemical analyses assessing phospho-ERK, activated caspase 3, and Ki-67 were performed as previously described ${ }^{19}$. Scoring was done by a pathologist, using the same parameters used for scoring human specimens.

\section{Supplementary Material}

Refer to Web version on PubMed Central for supplementary material.

\section{Acknowledgments}

This work is supported by the National Institutes of Health (CA122794, CA140594, CA137181, CA137008, CA147940, CA137008-01, 1U01CA141576, Lung SPORE P50CA090578), United against Lung Cancer Foundation, American Lung Association and Susan Spooner Research Fund.

\section{References}

1. Demetri GD, et al. Efficacy and safety of imatinib mesylate in advanced gastrointestinal stromal tumors. N Engl J Med. 2002; 347:472-480. [PubMed: 12181401]

2. Huang ME, et al. Use of all-trans retinoic acid in the treatment of acute promyelocytic leukemia. Blood. 1988; 72:567-572. [PubMed: 3165295]

3. Maemondo M, et al. Gefitinib or chemotherapy for non-small-cell lung cancer with mutated EGFR. N Engl J Med. 2010; 362:2380-2388. [PubMed: 20573926]

4. Mok TS, et al. Gefitinib or carboplatin-paclitaxel in pulmonary adenocarcinoma. N Engl J Med. 2009; 361:947-957. [PubMed: 19692680]

5. Ahrendt SA, et al. p53 mutations and survival in stage I non-small-cell lung cancer: results of a prospective study. J Natl Cancer Inst. 2003; 95:961-970. [PubMed: 12837832]

6. Gill RK, et al. Frequent homozygous deletion of the LKB1/STK11 gene in non-small cell lung cancer. Oncogene. 2011; 30:3784-3791. [PubMed: 21532627]

7. Ji H, et al. LKB1 modulates lung cancer differentiation and metastasis. Nature. 2007; 448:807-810. [PubMed: 17676035]

8. Nagata Y, et al. PTEN activation contributes to tumor inhibition by trastuzumab, and loss of PTEN predicts trastuzumab resistance in patients. Cancer Cell. 2004; 6:117-127. [PubMed: 15324695] 
9. Steels E, et al. Role of p53 as a prognostic factor for survival in lung cancer: a systematic review of the literature with a meta-analysis. Eur Respir J. 2001; 18:705-719. [PubMed: 11716177]

10. Yeh TC, et al. Biological characterization of ARRY-142886 (AZD6244), a potent, highly selective mitogen-activated protein kinase kinase 1/2 inhibitor. Clin Cancer Res. 2007; 13:1576-1583. [PubMed: 17332304]

11. Matsumoto S, et al. Prevalence and specificity of $L K B 1$ genetic alterations in lung cancers. Oncogene. 2007; 26:5911-5918. [PubMed: 17384680]

12. Weir BA, et al. Characterizing the cancer genome in lung adenocarcinoma. Nature. 2007; 450:893-898. [PubMed: 17982442]

13. Douillard JY, et al. Molecular predictors of outcome with gefitinib and docetaxel in previously treated non-small-cell lung cancer: data from the randomized phase III INTEREST trial. J Clin Oncol. 2010; 28:744-752. [PubMed: 20038723]

14. Mascaux $\mathrm{C}$, et al. The role of $R A S$ oncogene in survival of patients with lung cancer: a systematic review of the literature with meta-analysis. Br J Cancer. 2005; 92:131-139. [PubMed: 15597105]

15. Ji H, et al. Mutations in BRAF and KRAS converge on activation of the mitogen-activated protein kinase pathway in lung cancer mouse models. Cancer Res. 2007; 67:4933-4939. [PubMed: 17510423]

16. Verhoeven D, Teijaro JR, Farber DL. Pulse-oximetry accurately predicts lung pathology and the immune response during influenza infection. Virology. 2009; 390:151-156. [PubMed: 19493556]

17. Li D, et al. Bronchial and peripheral murine lung carcinomas induced by T790M-L858R mutant EGFR respond to HKI-272 and rapamycin combination therapy. Cancer Cell. 2007; 12:81-93. [PubMed: 17613438]

18. Dykes DJ, Bissery MC, Harrison SD Jr, Waud W. R Response of human tumor xenografts in athymic nude mice to docetaxel (RP 56976, Taxotere). Invest New Drugs. 1995; 13:1-11. [PubMed: 7499102]

19. Engelman JA, et al. Effective use of PI3K and MEK inhibitors to treat mutant Kras G12D and PIK3CA H1047R murine lung cancers. Nature Med. 2008; 14:1351-1356. [PubMed: 19029981]

20. Berghmans T, et al. Primary tumor standardized uptake value $\left(\mathrm{SUV}_{\max }\right)$ measured on fluorodeoxyglucose positron emission tomography (FDG-PET) is of prognostic value for survival in non-small cell lung cancer (NSCLC): a systematic review and meta-analysis (MA) by the European Lung Cancer Working Party for the IASLC Lung Cancer Staging Project. J Thorac Oncol. 2008; 3:6-12. [PubMed: 18166834]

21. Vansteenkiste JF, et al. Prognostic importance of the standardized uptake value on ${ }^{18}$ F-fluoro-2deoxy-glucose-positron emission tomography scan in non-small-cell lung cancer: an analysis of 125 cases. Leuven Lung Cancer Group. J Clin Oncol. 1999; 17:3201-3206. [PubMed: 10506619]

22. Carretero J, et al. Integrative genomic and proteomic analyses identify targets for $L \mathrm{kbl}$-deficient metastatic lung tumors. Cancer Cell. 2010; 17:547-559. [PubMed: 20541700]

23. Hainsworth JD, et al. A phase II, open-label, randomized study to assess the efficacy and safety of AZD6244 (ARRY-142886) versus pemetrexed in patients with non-small cell lung cancer who have failed one or two prior chemotherapeutic regimens. J Thorac Oncol. 2010; 5:1630-1636. [PubMed: 20802351]

24. Erasmus JJ, Rohren E, Swisher SG. Prognosis and reevaluation of lung cancer by positron emission tomography imaging. Proc Am Thorac Soc. 2009; 6:171-179. [PubMed: 19349485]

25. Singh M, et al. Assessing therapeutic responses in Kras mutant cancers using genetically engineered mouse models. Nature Biotechnol. 2010; 28:585-593. [PubMed: 20495549]

26. Tuveson D, Hanahan D. Translational medicine: cancer lessons from mice to humans. Nature. 2011; 471:316-317. [PubMed: 21412332]

27. Politi K, Pao W. How genetically engineered mouse tumor models provide insights into human cancers. J Clin Oncol. 2011; 29:2273-2281. [PubMed: 21263096]

28. Nishino M, et al. CT tumor volume measurement in advanced non-small-cell lung cancer: Performance characteristics of an emerging clinical tool. Acad Radiol. 2011; 18:54-62. [PubMed: 21036632]

29. Zhao B, et al. A pilot study of volume measurement as a method of tumor response evaluation to aid biomarker development. Clin Cancer Res. 2010; 16:4647-4653. [PubMed: 20534736] 
30. LoRusso PM, et al. Phase I and pharmacokinetic study of lapatinib and docetaxel in patients with advanced cancer. J Clin Oncol. 2008; 26:3051-3056. [PubMed: 18565892] 
a
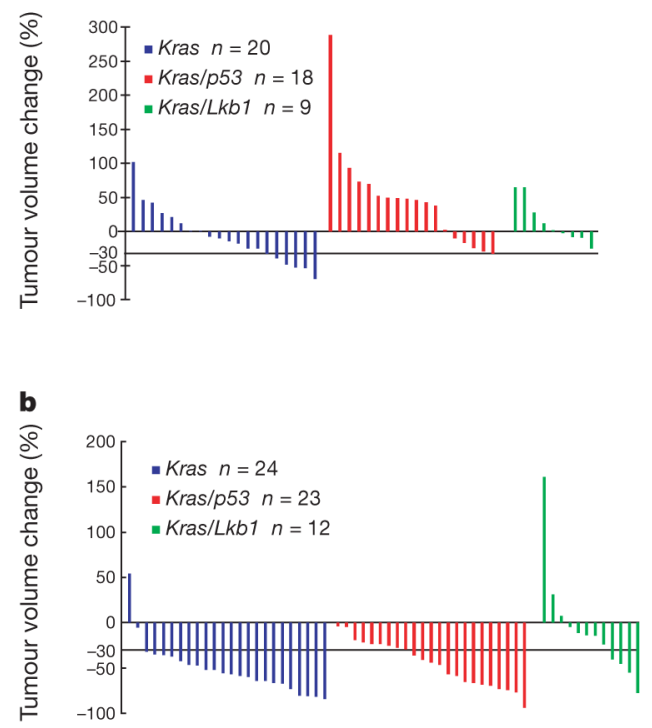

c

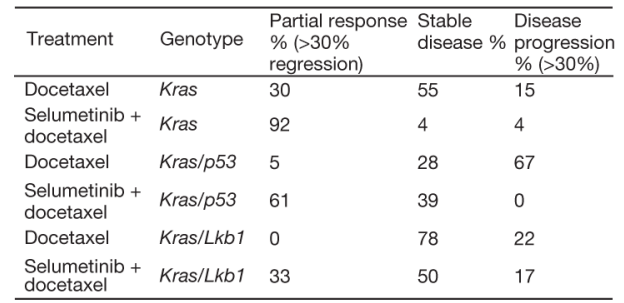

d
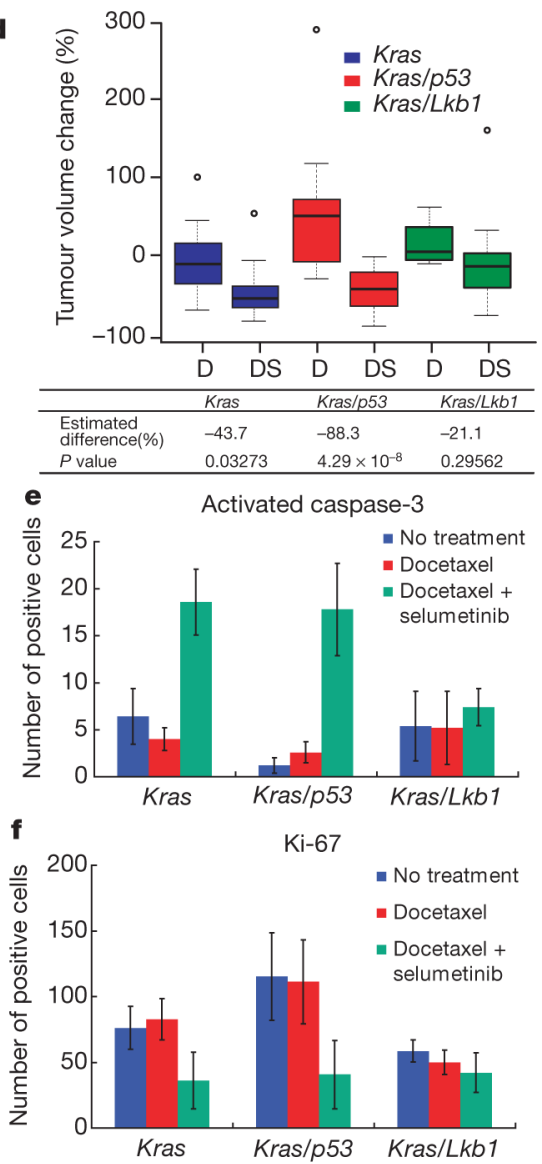

Figure 1. Docetaxel and selumetinib combination therapy is more efficacious than docetaxel monotherapy in Kras and $\mathrm{Kras} / \mathrm{p} 53$ lung cancers

a, Waterfall plot showing tumour response after 2 weeks of docetaxel treatment at $16 \mathrm{mg}$ $\mathrm{kg}^{-1}$ every 2 days. Each column represents one individual mouse, with data expressed relative to the pre-treatment tumour volume. $\mathbf{b}$, Waterfall plot showing tumour response after 2 weeks of docetaxel treatment at $16 \mathrm{mg} \mathrm{kg}^{-1}$ every 2 days in combination with daily selumetinib at $25 \mathrm{mg} \mathrm{kg}^{-1}$. c, Response rate of docetaxel and selumetinib combination therapy and docetaxel only in mice bearing tumours with different genotypes. d, Box plot showing tumour response for different genotypes with either docetaxel monotherapy (D) or combination treatment (DS). Lines depict median response, small circles indicate outliers. Estimated magnitude of difference between single and combination treatment within each genotype and corresponding one-sided $P$ values obtained by likelihood ratio test. e, $\mathbf{f}$, Number of activated caspase-3 (e) and Ki-67 (f) positive cells per microscopic field in mice of different genotypes after short-term treatment. Data represent the average of 5 different fields \pm standard deviation (s.d.) from 1-3 different mice (see Supplementary Table 6 for detailed information). 

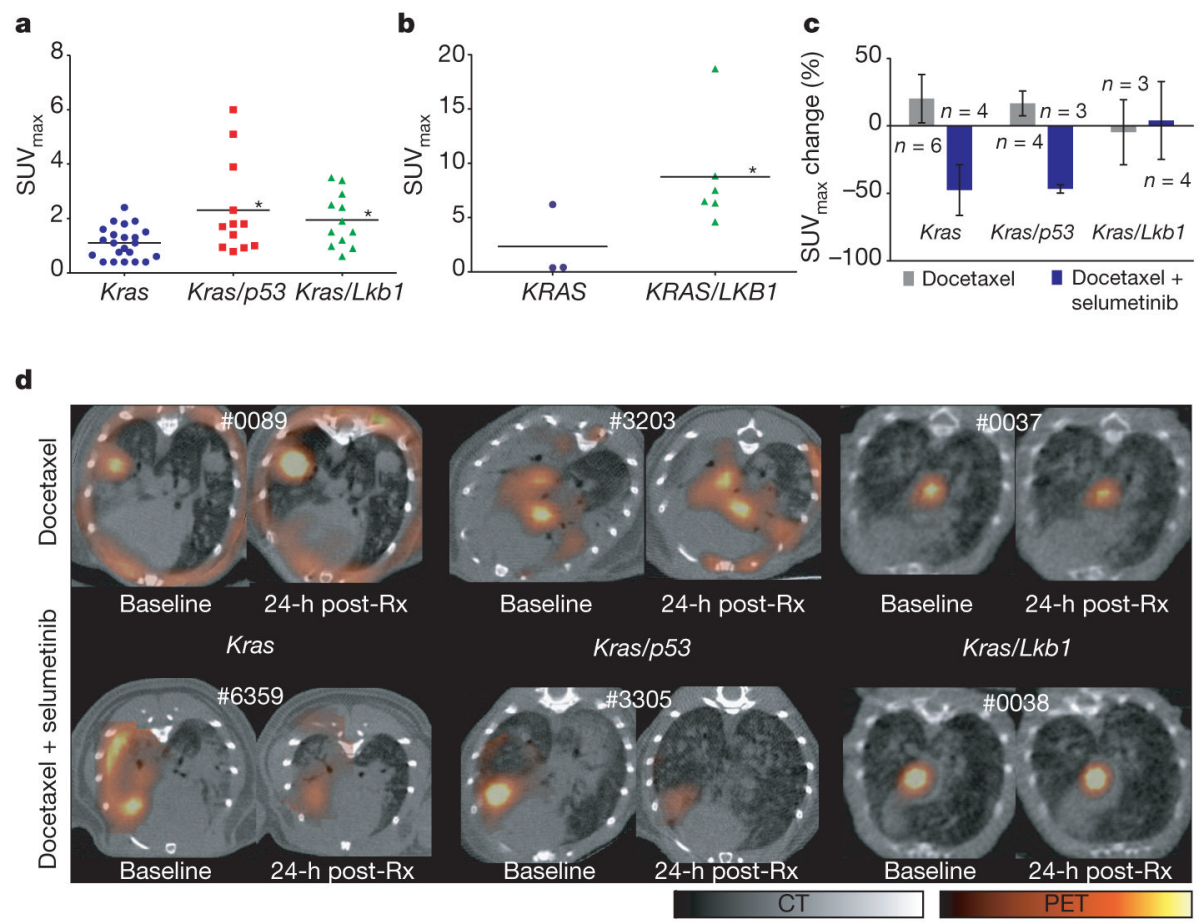

Figure 2. FDG-PET predicts treatment response

a, FDG-PET signal intensity $\left(\mathrm{SUV}_{\max }\right)$ in Kras, Kras/p53 and Kras/Lkb1 mutant mice. Statistical significance determined by rank sum test, with $* P<0.05$ for Kras compared to Kras/p53 mutant mice $(P=0.019)$, and Kras compared to Kras/Lkb1 mutant mice $(P$ $=0.014)$. b, FDG-PET signal intensity in patients with $K R A S$ or $K R A S / L K B 1$ mutant tumours. Statistical significance determined by two-sided Wilcoxon with $* P=0.048$. c, Comparisons of changes in FDG uptake by PET imaging after 1 day of treatment. Data are represented as mean \pm s.d. d, Representative FDG-PET/CT images of mice from different genotypes at baseline and 1 day after initiation of treatment. For each animal, the baseline and post-treatment (post-Rx) PET images are depicted with identical scales. The pseudocoloured FDG-PET images are fused with the grey-scale cross-sectional CT images. 


\section{a}
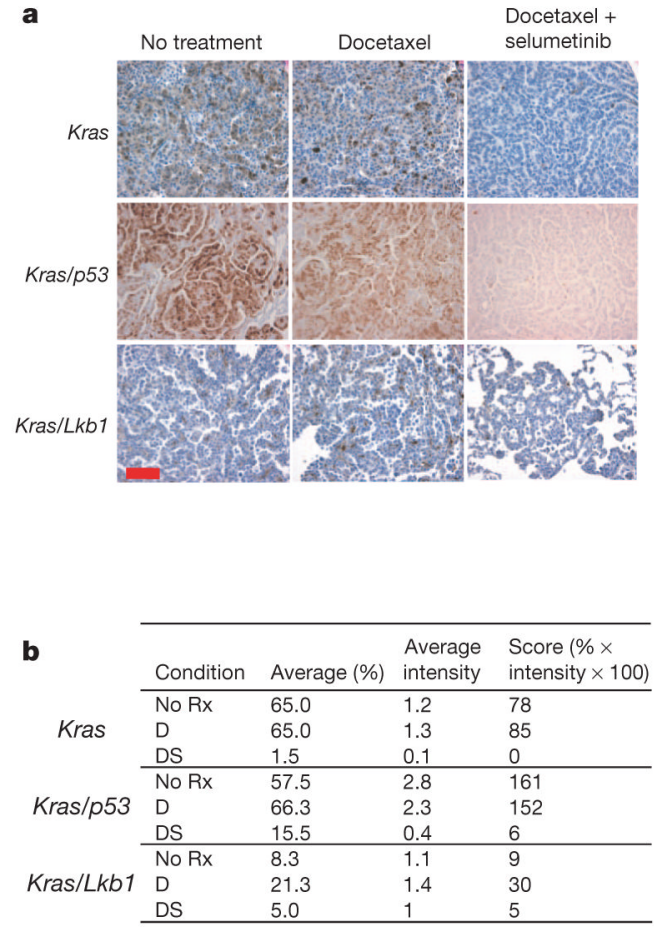

c

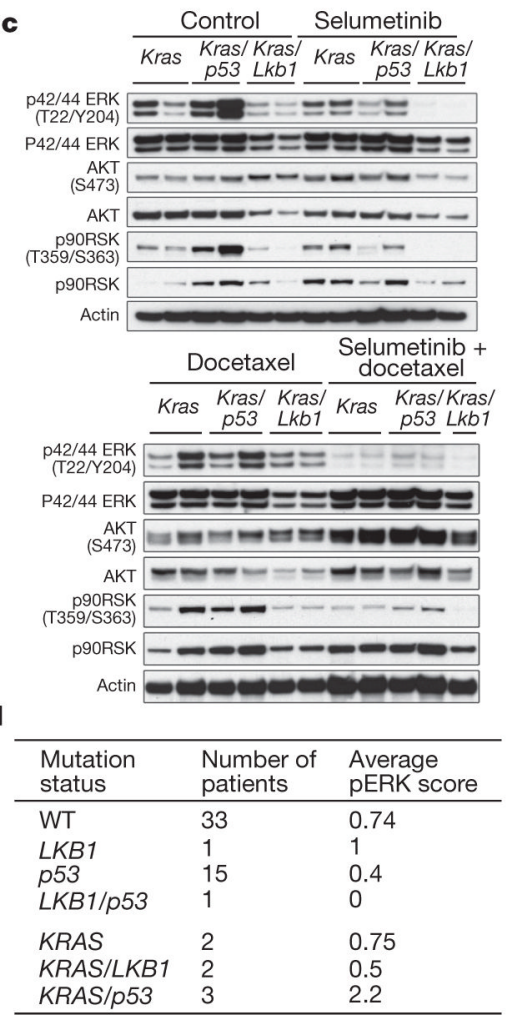

Figure 3. Modulation of the MEK-ERK pathway in response to treatment is different across the three genotypes

a, Immunostaining of phospho-ERK before and after treatment with docetaxel alone or in combination with selumetinib. Scale bar, $50 \mu \mathrm{m}$. b. Pathology score of phospho-ERK staining of mouse tumours shown in a. Both percentage of phospho-ERK positive cells and average intensity of phospho-ERK staining were scored for individual nodules, with a composite score derived by multiplying phospho-ERK positive percentage and average intensity. All samples were stained in the same batch. D, docetaxel; DS, docetaxel plus selumetinib; No Rx, untreated. c, Mice were subjected to treatment (two doses in $24 \mathrm{~h}$ ) as indicated, and killed $3 \mathrm{~h}$ after the second dose. Western blot was used to analyse tumour lysates with the indicated antibodies. d, Human NSCLC patients grouped by mutation status as indicated in the first column. Mean phospho-ERK (pERK) score from immunostaining is shown for each subset. 

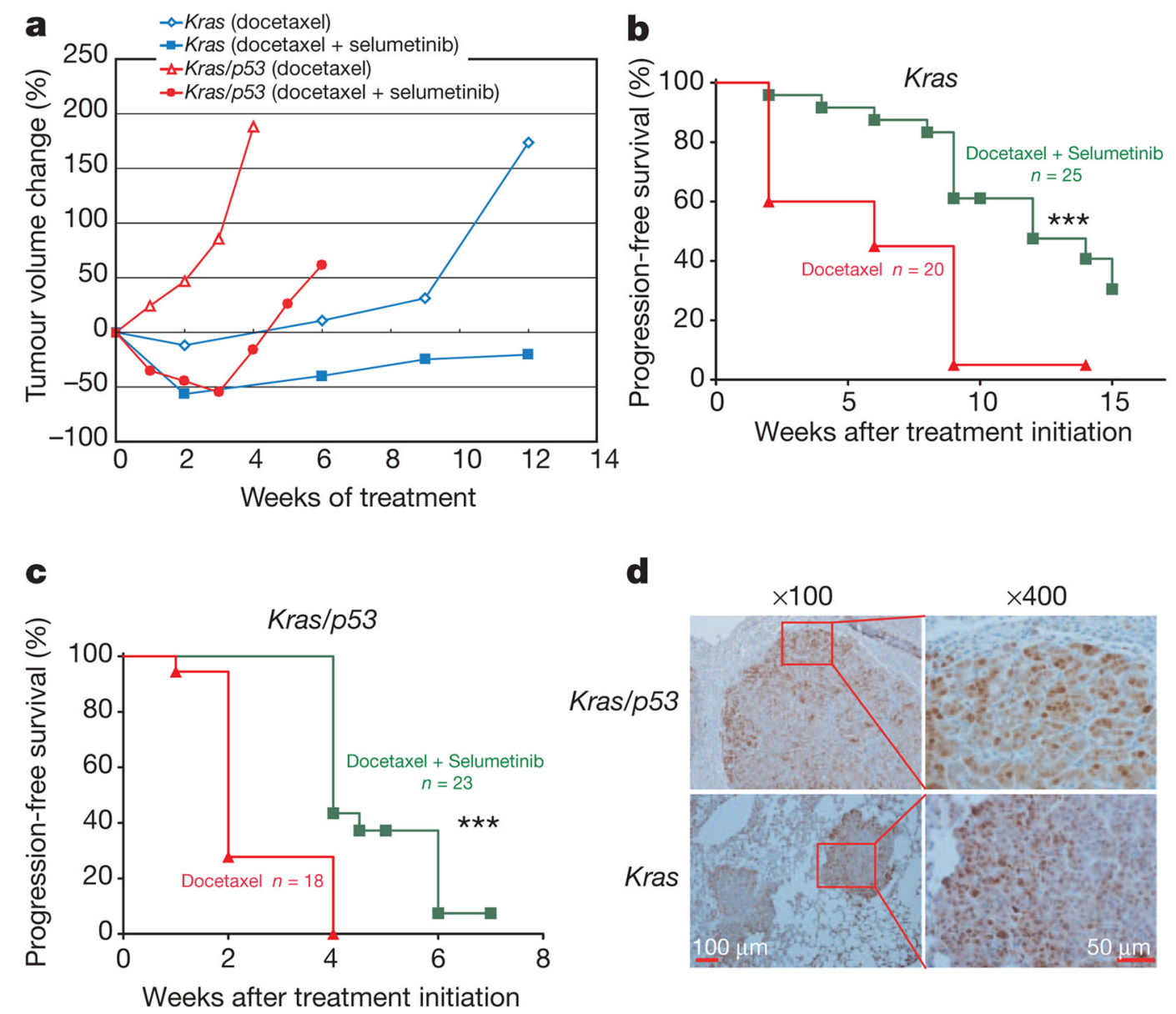

Figure 4. Long-term treatment outcome in Kras and Kras/p53 mice

a, Tumour volume was longitudinally assessed by MRI imaging in Kras and Kras/p53 mice treated with either docetaxel or docetaxel plus selumetinib. Data points represent median tumour volume relative to start of treatment for all available data the indicated time point. b, Progression-free survival for Kras mice treated with either docetaxel or docetaxel plus selumetinib. Median survival for single and combination treatments was 6 weeks and 12 weeks respectively, with $* * * P=0.0003$ by log-rank test. c, Progression-free survival for Kras/p53 mice treated with either docetaxel or docetaxel plus selumetinib. Median survival for single and combination treatments was 2 weeks and 4 weeks, respectively, with *** $P$ $<0.0001$ by log-rank test. Progression was defined as the time point when total tumour volume exceeded the baseline volume. d, Immunostaining of activation-specific phosphoERK of tumours from Kras/p53 and Kras mice with acquired resistance to docetaxel and selumetinib treatment. 